\title{
How to perform ostial stenting without contrast administration
}

\author{
Jerzy Sacha \\ Department of Cardiology, Faculty of Physical Education and Physiotherapy, Opole University of Technology, Opole, Poland
}

Adv Interv Cardiol 2020; 16, 1 (59): 107-109

DOI: https://doi.org/10.5114/aic.2020.93918

Perfect ostial stenting may constitute a challenge during percutaneous coronary intervention $(\mathrm{PCl})$ and it usually involves several contrast injections during stent positioning. The presented images demonstrate a technique which improves precision of ostial stenting and it also enables the procedure to be performed without contrast administration. This method may be employed in any intervention but it is particularly helpful during zero-contrast $\mathrm{PCl}$, which is a new approach for prevention of contrast-induced acute kidney injury [1-3].

In the presented case, a 78-year-old man required ostial stenting of a significant left main stenosis - Figures $1 \mathrm{~A}, \mathrm{~A}^{\prime}$ show the left coronary angiography and corresponding intravascular ultrasound (IVUS) image of the

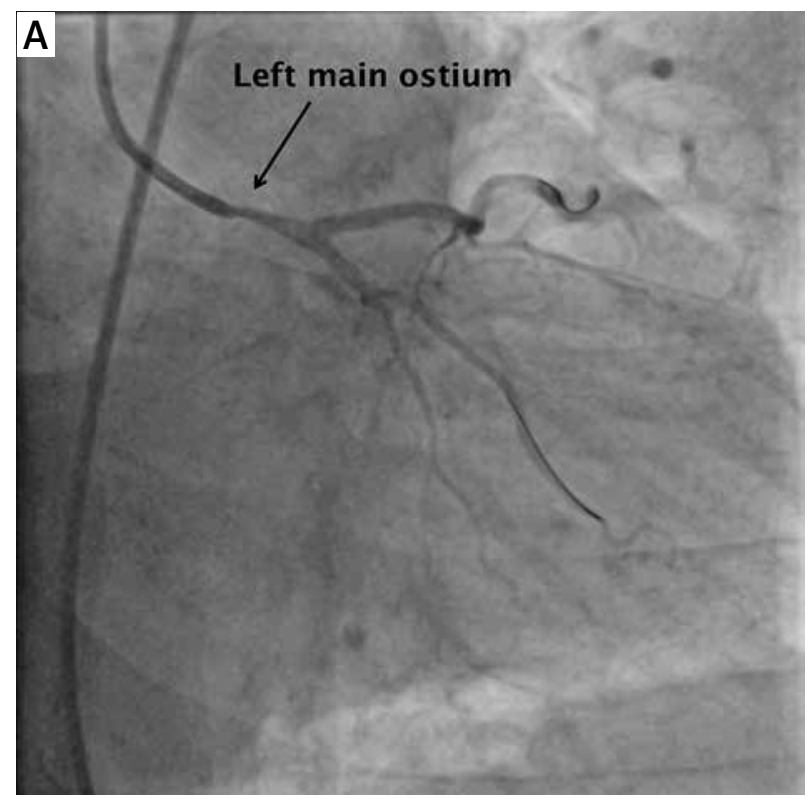

ostial lesion (after balloon pre-dilation). Of note, there was no landmark (e.g. calcification) within the ostium which might indicate a proximal landing zone for stent implantation. To perform ostial stenting under IVUS guidance, two guidewires should be inserted using a $7 \mathrm{Fr}$ guide catheter; the first wire is for the balloon and stent delivery, while the second one is for the insertion of the IVUS probe. Following the stent insertion, the IVUS probe should be positioned in such a way that its active tip corresponds to the proximal stent marker. Then, both devices ought to travel as one unit to the level of the coronary ostium; i.e. IVUS imaging should show the border between the ostium and the aorta (Figures 1 B, B'). Importantly, after partial stent deployment (Figures 1 C, C'),

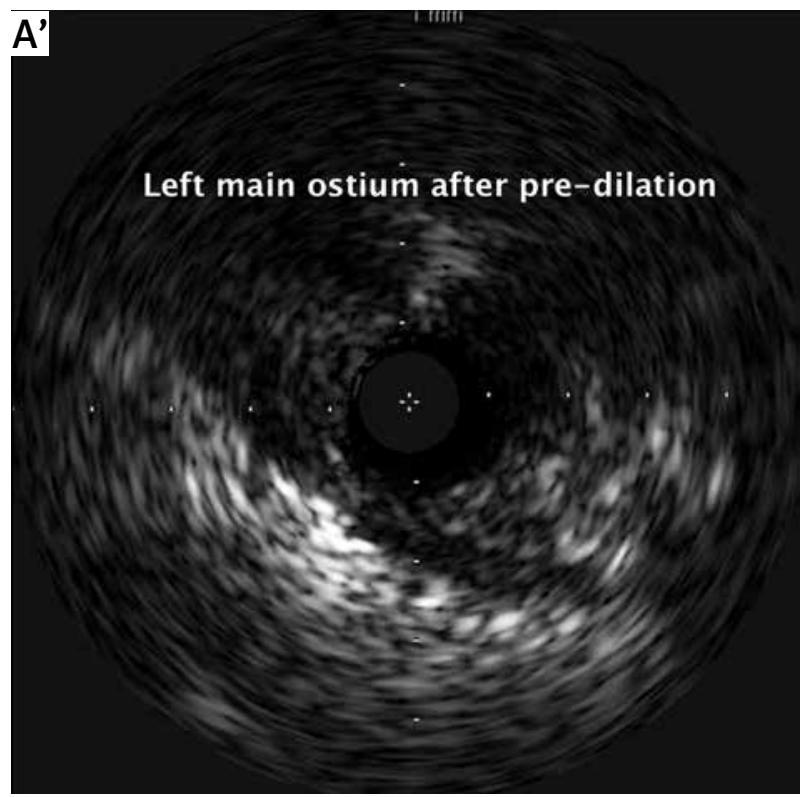

Figure 1. Angiographic pictures and corresponding intravascular ultrasound (IVUS) images for the left main ostial stenting. A, A' - The left coronary angiography and the matching IVUS image of the ostial lesion (after balloon pre-dilation)

\section{Corresponding author:}

Jerzy Sacha MD, PhD, Department of Cardiology, Faculty of Physical Education and Physiotherapy, Opole University of Technology, Opole, Poland, phone: +48 7745206 60, e-mail: sacha@op.pl

Received: 1.10.2019, accepted: 18.10.2019. 

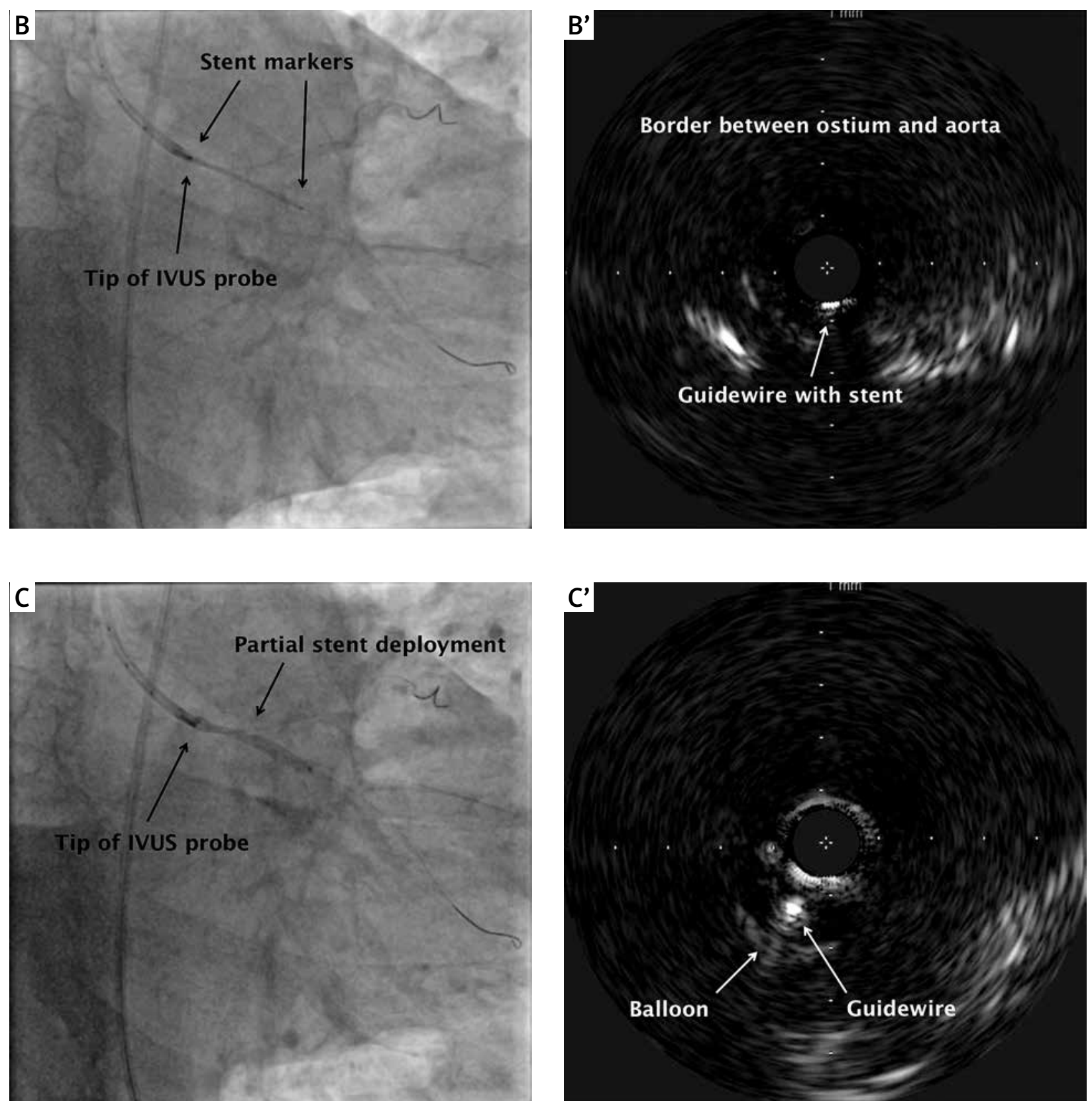

Figure 1. Cont. B, B' - Stent positioning with the help of IVUS imaging (the active tip of the IVUS probe corresponds to the proximal stent marker). C, C' - Partial stent deployment - importantly, after the partial deployment, the balloon must be deflated and the IVUS probe has to be retracted to prevent the probe being trapped by the stent 

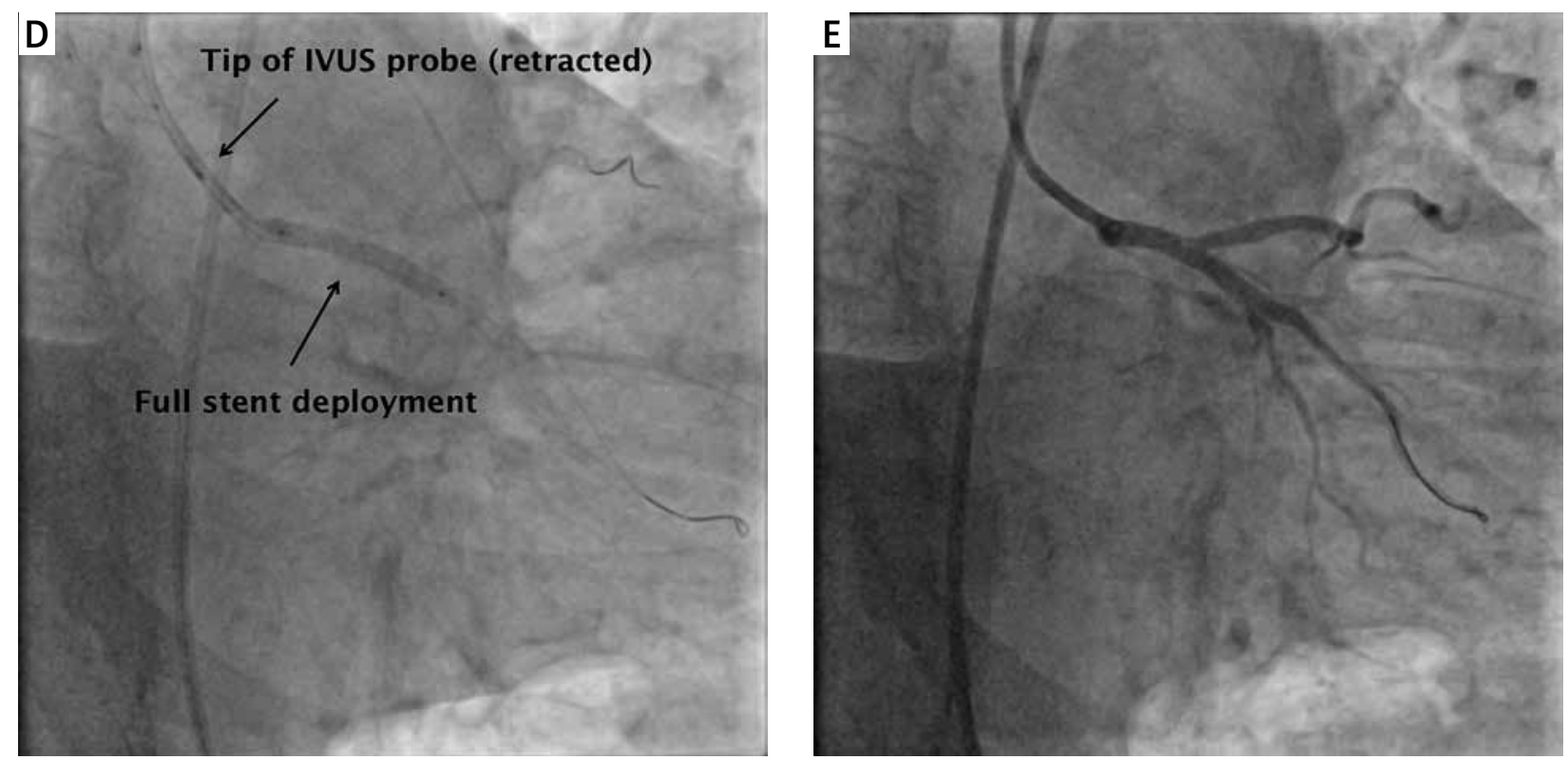

Figure 1. Cont. D - Full stent deployment (note that the IVUS probe is retracted to the guide). E - Final stenting result

the balloon must be deflated and the IVUS probe has to be retracted to prevent its plastic tip being trapped by the stent - only then can the stent be fully deployed (Figure $1 \mathrm{D})$. The IVUS probe and the corresponding guidewire should be withdrawn and the final check with IVUS imaging may be performed by insertion of the IVUS probe over the first wire. The whole system, i.e. the $7 \mathrm{Fr}$ guide catheter, two guidewires, stent and IVUS probe, is very stable and enables precise stent deployment (Figure $1 \mathrm{E}$ ). This simple technique is helpful in any ostial stenting, but it is particularly useful in cases where stent positioning within the ostium is challenging (e.g. due to acute angle take-off of the coronary artery) and in patients with severe renal dysfunction, since it enables the procedure to be performed without contrast usage.

\section{Conflict of interest}

The author declares no conflict of interest.

\section{References}

1. Ali ZA, Karimi Galougahi K, Nazif T, et al. Imaging- and physiology-guided percutaneous coronary intervention without contrast administration in advanced renal failure: a feasibility, safety, and outcome study. Eur Heart J 2016; 37: 3090-5.

2. Sacha J, Gierlotka M, Feusette P, et al. Ultra-low contrast coronary angiography and zero- contrast percutaneous coronary intervention for prevention of contrast-induced nephropathy: step-by-step approach and review. Adv Interv Cardiol 2019; 15: 127-36.

3. Sacha J, Gierlotka M, Lipski P, et al. Zero-contrast percutaneous coronary interventions to preserve kidney function in patients with severe renal impairment and hemodialysis subjects. Adv Interv Cardiol 2019; 15: 137-42. 